\title{
The Effect of Velocity of Nodes on the Performance of Routing Protocols in Mobile Ad-hoc Networks
}

\author{
Zubair lqbal \\ Asst. Prof., Dept. of CS \& IT, \\ Moradabad Institute of Technology \\ Moradabad, U.P., India
}

\author{
Prachi Gupta \\ Asst. Prof., Dept. of CS \& IT, \\ Moradabad Institute of Technology \\ Moradabad, U.P., India
}

\begin{abstract}
MANET $[1,2]$ is an autonomous system in which mobile hosts connected by wireless links [3] are free to move randomly [4] and often act as routers at the same time. Node failures and arbitrary movement of nodes break the routes and lead the frequent operation of rebuilding routes that consume lots of the network resources and the energy of nodes. This means we need a routing protocol that can enhance the network stability. There are three main categories of routing protocols: Proactive, Reactive and Hybrid. In this paper the performance of one proactive protocol (DSDV) and two reactive protocols (DSR and AODV) [5] has been evaluated. This evaluation is done by network Simulator 2(NS2). The performance is measured by means of following metrics: Packet Delivery Ratio, Average End to End Delay, and Normalized Routing Load.
\end{abstract}

\section{General Terms}

MANET

\section{Keywords}

MANET, DSDV, DSR, AODV, NS-2, PDF, and NRL.

\section{INTRODUCTION}

A mobile ad hoc network (MANET) is a self-configuring infrastructure less network of mobile devices connected by wireless links. Each device in a MANET is free to move independently in any direction, and will therefore change its links to other devices frequently. Each must forward traffic unrelated to its own use, and therefore be a router. The primary challenge in building a MANET is equipping each device to continuously maintain the information required to properly route traffic. The problem of maintaining route increases as the velocity of these independently moving node increases. The nodes participating in scenario are also used as router at the same time. Thus it is the hardest job to find the stable route between source and destination and also the sequence of node between source and destination. Thus we are always in need of a best suited protocol for the scenario. There is a lot of work done on evaluating performances of various MANET routing protocols. In this paper we have tried to evaluate the performance of one proactive protocol (DSDV) and two reactive protocols (DSR and AODV). This simulation has done by means of NS-2 [6,7] which is a discrete event simulator developed at Berkeley University.
The rest of this paper is organized as follows. Section 2 briefly described the routing protocols that are evaluated. Section 3 presents the Simulation environment used for evaluation of the said protocols. Section 4 presents the simulation results and observations. Finally, section 5 concludes the paper.

\section{MANET ROUTING PROTOCOLS}

This section briefly described the protocols considered for the simulation that are DSDV, DSR and AODV.

\subsection{DSDV Protocol}

In Destination Sequenced Distance Vector routing (DSDV) [8], each node maintains a routing table wherein the next hop information for each reachable destination is maintained. Every node in the network periodically broadcasts its routing table with monotonically increasing sequence numbers. An update is done using the Bellman-Ford algorithm. A broken link can be detected if no broadcasts have been received from a node for a while. On detection of a broken link, all routes passing through that hop are assigned an "infinity metric".

\subsection{DSR Protocol}

Dynamic Source Routing (DSR) [9, 10] employs "source routing" wherein the source determines the complete sequence of nodes through which a packet has to be routed. Whenever a source has a packet to transmit, it checks its routing table for a route to the destination. In case a route is not found, a route request (RREQ) broadcast is initiated. On receiving this request, each node again broadcasts this request by appending its address to the request packet until this packet reaches the destination. The destination replies to the first request that reaches it. It sends a route reply (RREP) to the source containing the route from the source to the destination. When this packet reaches the source, a connection is established and all subsequent packets contain the complete route in the packet header. No routing information is maintained at the intermediate nodes. When the data link layer at a particular node encounters a transmission failure, it issues an error notification to the source and a new route search is initiated. 


\subsection{AODV Protocol}

Ad-hoc On-demand Distance Vector [11] routing algorithm borrows its salient features from DSR and DSDV. When a source needs a path to a destination, it broadcasts a route request message enclosing a monotonically increasing "broadcast id" and the last known sequence number to that destination. The route request is broadcast until it reaches a node that has a route to the destination with a destination sequence number higher than that enclosed in the request. A route request propagating through the network establishes the next hop information for the reverse route to the source. A route reply generated by the destination propagates along the reverse route and establishes the forward route information at the intermediate nodes. Each node records only the next hop for a destination and not the entire route as done in source routing protocols. Routing table information in AODV is restricted to the active modes. A neighbor is considered active if it originates or relays at least one packet for the destination within the most recent "active timeout" period. Failure of a link can be detected via hello messages or link layer detection. When a link goes down, the upstream nodes are notified of the failure and that destination is marked as unreachable in the routing tables of these nodes.

\section{SIMULATION ENVIRONMENT}

Network simulator ns 2.35 were used for simulation, most widely used network simulator and freely available. This research work uses three performance Evaluation parameters: Packet Delivery Ratio, Average End to End Delay, and Normalized Routing Load. The parameters for the simulation are as follows:

Table 1. Simulation Parameters

\begin{tabular}{|c|c|}
\hline Parameter & Value \\
\hline Transmission range & $250 \mathrm{~m}$ \\
\hline Simulation time & $700 \mathrm{~s}$ \\
\hline Topology size & $500 \mathrm{~m} \times 500 \mathrm{~m}$ \\
\hline Number of mobile nodes & 10 \\
\hline Traffic type & constant bit rate (CBR) \\
\hline Packet size & Variable \\
\hline Maximum speed & \\
\hline
\end{tabular}

\section{SIMULATION RESULTS AND OBSERVATIONS}

This section presents simulation observations that compare the performance of the protocols described in section 2 .

\subsection{Packet Delivery fraction (PDF)}

Fig 1 shows packet delivery ratio with speed of nodes varying from 1 to 250 for DSDV, AODV and DSR routing protocols. The red line shows graph for AODV, the blue line shows graph for DSR and the green line shows the graph for DSDV protocol.
The delivery ratio for AODV and DSR protocols is always greater than 90 percent because these are reactive protocols and hence numbers of received packets are more. For DSDV, the delivery ratio is decreasing with increase in speed because it is proactive and numbers of received packets are less than AODV and DSR. But generally the graph for the DSR protocol lies above than that of DSDV for most cases. However in certain cases the DSDV protocols is also better.It is more likely for the mobile nodes to have fresher and shorter routes to a gateway and thereby minimizing the risk for link breaks. Link breaks can result in lost data packets since the source continues to send data packets until it receives a RERR message from the mobile node that has a broken link. The longer the route is (in number of hops), the longer times it can take before the source receive a RERR and hence more data packets can be lost.The packet delivery ratio should be high. In this analysis, AODV and DSR show the maximum packet delivery ratio.

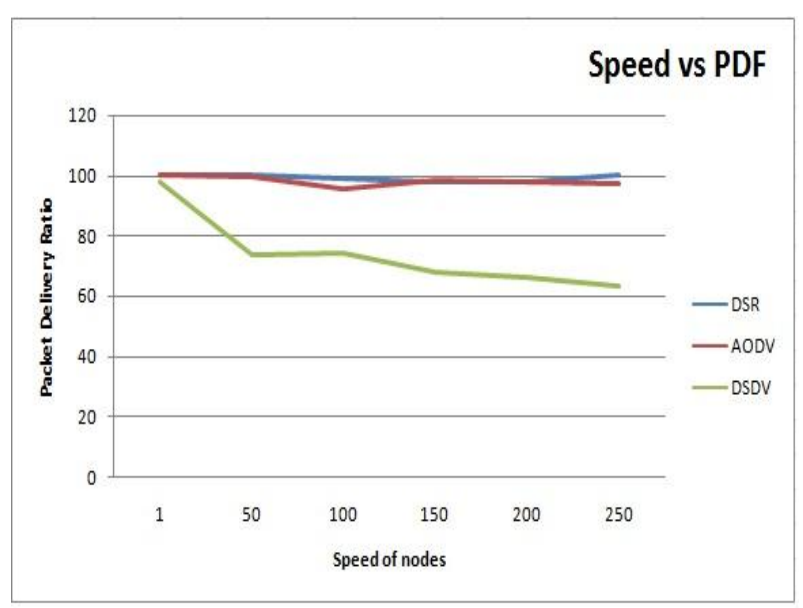

Fig 1: Speed verses Packet delivery ratio

\subsection{Average End-To-End Delay}

Fig 2 shows that the average end-to-end delay is least for the DSDV approach, then for AODV approach and highest for the DSR approach. The reason is that the periodic gateway information sent by the gateways allows the mobile nodes to update their route entries for the gateways more often, resulting in fresher and shorter routes. With the DSR (reactive approach) a mobile node continues to use a route to a gateway until it is broken. In some cases this route can be pretty long (in number of hops) and even if the mobile node is much closer to another gateway it does not use this gateway but continues to send the data packets along the long route to the gateway further away until the route is broken. Therefore, the end-to-end delay increases for these data packets resulting in increased average end-to-end delay for all data packets. The average end-to-end delay is decreased slightly for short pause time intervals when the advertisement interval is increased. At the first thought this might seem unexpected. However, it can be explained by the fact that very short advertisement intervals result in a lot of control traffic which lead to higher processing times for data packets at each node.The end-to-end delay should be low. In this analysis DSDV shows the minimum end-to-end delay. 


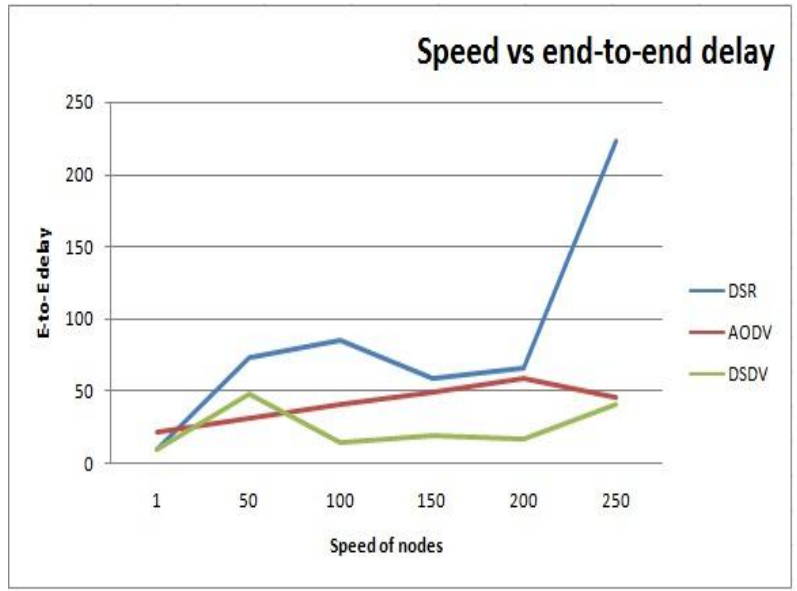

Fig 2: Speed verses end-to-end delay

\subsection{Normalized Routing Load (NRL)}

Figure 3 shows normalized routing load with speed of nodes varying from 1 to 250 for DSDV, AODV and DSR routing protocol. The red line shows graph for AODV, the blue line shows graph for DSR and the green line shows the graph for DSDV protocol. For DSDV, the NRL is increasing with increase in speed because it is proactive and no of received routing packets are less than data packets. For AODV, the NRL is increasing with increase in speed because no of received routing packets are less than data packets. For DSR, the NRL is decreasing with increase in speed because no of received routing packets are more than data packets.

The NRL ratio should be low. In this analysis, DSR shows the minimum normalized routing load.

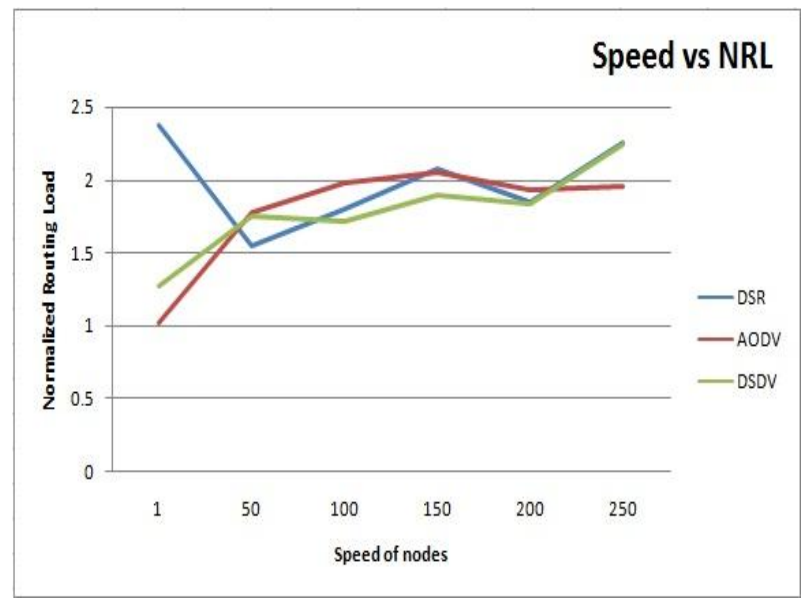

Fig 3: Speed verses normalized routing load

\section{CONCLUSION}

The results of the simulation indicate that performance of the DSR protocol is superior to standard DSDV. It is also observed that the performance is better especially when the pause time is low. For higher pause time although DSR is better for most cases but their delivery ratio remains close to each other.
The packet delivery ratio must be high. In this analysis, AODV and DSR showed the maximum packet delivery ratio. The end-to-end delay must be low. This analysis shows DSDV have minimum end-to-end delay. The NRL ratio must be low. In this analysis DSR shows the minimum normalized routing load.It is also true that any of the single protocol does not supersede the other one. Their performance depends upon the different scenarios.

\section{FUTURE SCOPE}

This research can be extended by including other protocols and more performance evaluation parameters. This paper only focused on one proactive (DSDV) and two reactive (DSR, AODV) routing protocols. However there are many other protocols like TORA, OLSR, and ZRP that could be subject to further studies.

\section{REFERENCES}

[1] M.S. Corson, J. Macker, Mobile ad hoc networking: routing protocol performance issues and evaluation considerations. Internet RFC 2501, January 1999, http://www.ietf.org/rfc/rfc2501.txt.

[2] M.S. Corson, J.p. Macker, G.H. Cirincione, Internetbased mobile ad hoc networking, IEEE internet computing 3 (4) (1999) 63-70.

[3] C. K. Toh: Maximum battery life routing to support ubiquitous mobile computing in wireless adhoc networks, Communications Magazine, IEEE, Vol. 39, 2001, pp 138-147.

[4] Mobile Ad-hoc Networks (MANET) http://www.ietf.org/html.charters/manetcharter.html. (1998-11-29)

[5] E. M. Royer and C. K. Toh: A review of current routing protocols for ad hoc mobile wireless networks, IEEE Personal Communications, 6(2), April 1999, pp 46-55; Proceedings of IEEE ICC'98, August 1998, pp 156-160.

[6] Matthias Transier "Ns2 tutorial running simulations".

[7] www.isi.edu/nsnam/ns/tutorial Marc Greis tutorial on ns2.

[8] C.E. Perkins \& P. Bhagwat, "Highly Dynamic Destination Sequence-Vector Routing (DSDV) for Mobile Computers", Computer Communication Review, vol. 24, no.4, 1994, pp. 234-244.

[9] David B. Johnson and David A.Maltz: Dynamic source routing in ad hoc wireless networks, In Mobile Computing, edited by Tomasz Imielinski and Hank Korth, chapter 5, Kluwer Academic Publishers, pp 153 181.

[10] Yasser Kamal Hassan, Mohamed Hashim Abd El-Aziz, and Ahmed Safwat Abd El-Radi: Performance Evaluation of Mobility Speed over MANET.

[11] C.E. Perkins and E.M. Royer, "Ad-Hoc on-Demand Distance Vector Routing," Proc. Workshop Mobile Computing Systems and Applications (WMCSA '99), Feb. 1999 pp. 90-100. 